\title{
Uma Nova Abordagem para o Perfilamento de Usuários em Ambientes de Aprendizagem Ubíqua
}

\author{
Argemiro S. de Aliança Neto ${ }^{1}$, Francisco M. Mendes Neto ${ }^{1}$, Jonathan D. C. Moreira ${ }^{1}$ \\ ${ }^{1}$ Programa de Pós-graduação em Ciência da Computação (PPgCC) \\ Universidade Federal Rural do Semi-Árido (UFERSA) \\ Av. Francisco Mota, 572. CEP 59.625-900. Costa e Silva, Mossoró - RN - Brasil \\ argemiront@gmail.com, \{miltonmendes, jdarlan\}@ufersa.edu.br
}

\begin{abstract}
Personalization became the key element to ubiquitous educational service offer. For the educational content to be provided properly for users, it's necessary that their profiles be known. Common approaches to capture profile include the use of ontologies and they are based only through the web browsing history. This paper presents a new approach to the capture of profiles while adding the contents available in the web sites and the ones made by users through the profile analyses process.
\end{abstract}

Resumo. Personalização tornou-se o elemento chave na oferta de serviços educacionais ubíquos. Para que conteúdos educacionais sejam fornecidos adequadamente para o usuário, é necessário que seu perfil seja conhecido. Abordagens comuns de captura de perfil incluem o uso de ontologias e são baseadas apenas no histórico de navegação. Este artigo apresenta uma nova abordagem para a captura dos perfis ao adicionar os conteúdos disponíveis nos sites e os produzidos pelos usuários no processo de análise do perfil.

\section{Introdução}

Personalização tornou-se um elemento chave para o sucesso dos mais diversos tipos de soluções da Web 2.0, em especial para ambientes de aprendizagem ubíqua [Vigneshwari e Aramudhan 2012]. A adequação do conteúdo educacional ao perfil do estudante é o foco de tais personalizações [Yarandi et al. 2012, Yau e Joy 2010, Taraghi 2012, Ferreira et al. 2012]. O conhecimento adequado e atualizado sobre o perfil do usuário, ou seja, seus interesses, suas crenças, experiências de vida, intenções e comportamentos na Web, oferece a estrutura básica para o funcionamento de tais sistemas [Peña et al. 2013, Karanasios et al. 2013].

A captura e manutenção eficaz do perfil do usuário não é uma atividade trivial. Um dos problemas mais comuns no processo de construção destes perfis está relacionado ao método de captura da informação. O perfil pode ser capturado de maneira implícita ou explícita [Reformat e Golmohammadi 2009]. A técnica explícita é a forma mais simples e direta de se obter as informações necessárias e pode ser feita com o preenchimento de um simples formulário. No entanto, esta abordagem apresenta limitações. O usuário pode não se sentir confortável em fornecer determinadas informações de interesse pessoal, comprometendo a eficácia desta técnica. No método implícito, dados da navegação na Web, localização, conteúdos das páginas visitadas, etc. são analisados em busca de um padrão que denote os interesses do usuário, atualizando-o automaticamente seu perfil. 
A utilização de ontologias em sistemas educacionais é uma abordagem com resultados relevantes [Gluz e Vicari 2010]. Ontologias facilitam o processo de construção dos perfis dos usuários [Yingchen et al. 2009, Yarandi et al. 2012, Gao et al. 2013, Sutterer et al. 2008, So et al. 2009, Rungworawut e Kachonsri 2012], porém os resultados apresentados ainda oferecem sérias limitações quanto à análise do conteúdo acessado e aplicabilidade em domínios e aplicações diferentes dos originais.

Este trabalho apresenta uma nova abordagem para a construção implícita de tais perfis de maneira genérica, independente do domínio de aplicação e facilmente adaptável a sistemas de recomendação de conteúdo. O restante deste artigo está organizado da seguinte forma: na seção 2 são elencados trabalhos relacionados à proposta; Na seção 3 é apresentada uma nova abordagem para o perfilamento de usuários em ambientes de aprendizagem ubíqua; Na seção 4 são discutidos os resultados da pesquisa; E, por fim, as conclusões e os trabalhos futuros são apresentados na seção 5.

\section{Trabalhos Relacionados}

Em [Reformat e Golmohammadi 2009] propõe-se um método baseado na técnica implícita para captura e atualização automática de perfis de usuários. O método analisa os dados dos $\log s$ de navegação em busca de termos relativos ao domínio em questão (música) e os compara com os registrados para o perfil do usuário. Para realizar tal comparação, o conceito de similaridade semântica é empregado. Após definida a similaridade, o método calcula o índice de importância com base na frequência dos acessos a um determinado recurso. A similaridade e importância são combinadas através de um método baseado em lógica fuzzy. Por fim, o termo é inserido no perfil do usuário através de uma instância da classe em questão, mesclando dados de domínio com os dos usuários.

Peña et al. (2013) apresentam uma técnica de construção automática de perfis de usuários com base nas URLs dos tweets públicos destes. Estas URLs são categorizadas de acordo com os dados dos websites DBPedia ${ }^{1}$ e OpenDNS ${ }^{2}$. Estas categorias são armazenadas em uma estrutura hierárquica de tags em uma base de dados local. Uma ontologia padrão de domínio é criada com base nesta estrutura e povoada com dados extraídos destas e de várias outras fontes. Ao serem baseadas em estruturas de dados nãocontroladas e por vezes variantes, as estruturas hierárquicas das ontologias também se tornam mutáveis no tempo. Esta variação acarreta em maior complexidade nos processos de análise, comparação e inferência sobre os dados armazenados.

Considerar apenas o tipo de página e a URL para definição do perfil é uma abordagem que apresenta limitações ao desconsiderar os conteúdos relacionados à pagina e os produzidos pelos usuários, definidos como digital traces. Dspotakis et al. (2011) apresentam um framework, chamado de ViewS, que estabelece os principais passos para captura dos digital traces. Apesar de evoluir ao considerar os digital traces, este ainda apresenta limitações por necessitar da intervenção explícita do usuário na captura do perfil.

Com o objetivo de contornar o problema da dependência do usuário em [Dspotakis

\footnotetext{
${ }^{1}$ DBPedia é resultado de um esforço comunitário com o objetivo de extrair informações estruturadas da Wikipedia (http: / / dbpedia.org).

${ }^{2}$ OpenDNS cloud websites tagging é um banco de dados mantido pela comunidade para classificação e marcação de sites de acordo com o seu conteúdo (http://community.opendns.com/ domaintagging/).
} 
et al. 2011], Karanasios et al. (2013) propuseram uma nova arquitetura para enriquecimento semântico de digital traces. O processo utilizado por esta arquitetura contém duas etapas. Na primeira, uma ontologia de domínio é analisada e as palavras-chaves são registradas em um ferramenta de busca semântica. Na segunda etapa, o texto a ser enriquecido é pesquisado em busca destas palavras-chaves. Quando uma correspondência é encontrada realiza-se uma anotação no texto com os dados da ontologia. Por fim, estas anotações são indexadas e armazenadas em uma base de dados semântica.

Este artigo propõe uma nova abordagem baseada na arquitetura definida em [Karanasios et al. 2013] para a criação e representação do perfil do usuário com base não somente nas URLs acessadas, mas também nos conteúdos das páginas, em ontologias e, por fim, nos digital traces produzidos pelos usuários. Para isto, é proposto o Semantic Augmentation System (SAS), um sistema de enriquecimento semântico de conteúdos associados a perfis de usuários em forma de biblioteca, que utilizada uma UPO (do inglês User Profile Ontology) básica que mantém dados dos usuários e links para os conceitos das ontologias de domínio empregadas no sistema. A ser empregado como ferramenta de perfilagem no ambiente de aprendizagem ubíqua MobiLE+ [Jácome Jr 2014].

\section{Abordagem Proposta}

O perfil do usuário representa principalmente seus interesses, identificáveis a partir dos conteúdos dos sites acessados. Porém, não necessariamente o usuário tem os mesmos níveis de interesse para todos os assuntos, tampouco os conteúdos dos sites são igualmente distribuídos e uniformes. Para que estes sejam conhecidos, duas questões são observadas: (i) qual a relação entre um determinado conteúdo e um domínio? E, (ii) qual o nível de interesse de um usuário em um domínio específico? Estas questões são avaliadas a partir de informações armazenadas em uma ontologia de perfil (UPO) e relacionadas através da similaridade semântica entre os pares.

Denominada de SASProfile, a UPO é utilizada pelo Indexador Semântico, que realiza a indexação das anotações semânticas realizadas por um Analisador Semântico. Esta arquitetura é detalhada na Figura 1.

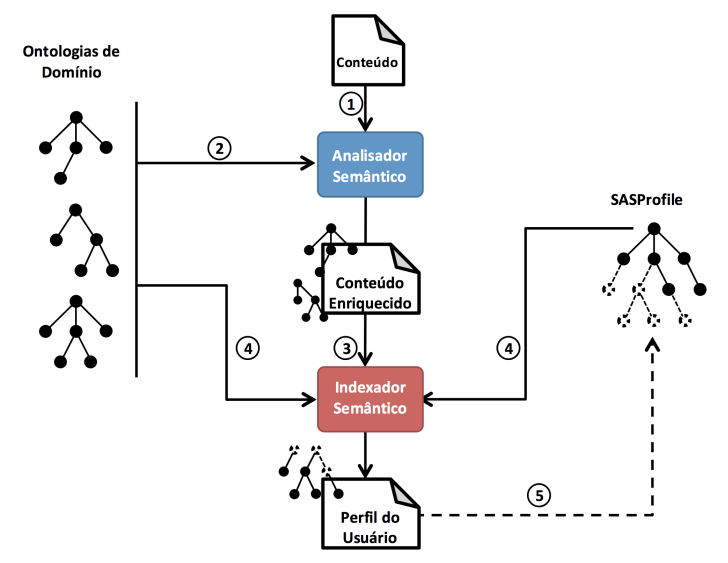

Figura 1. Arquitetura do Sistema

No momento em que um conteúdo (site, vídeo, rede social, etc.) é acessado, o componente Analisador Semântico identifica e isola as anotações e/ou marcações já existentes, como HTML e XML (passo 1). O conteúdo restante é semanticamente anotado 
com conceitos provenientes das ontologias de domínio (passo 2). Os dados das anotações semânticas do conteúdo sendo enriquecido são transmitidos para o Indexador Semântico (passo 3), que seleciona dados importantes com base nos já cadastrados na UPO (passo 4). Os dados tratados são indexados e armazenados na SASProfile para o perfil individual do usuário (passo 5). O Indexador Semântico também fornece as respostas para os questionamentos sobre a relação entre um conteúdo e um domínio específico e o interesse de um dado usuário para com este domínio.

\subsection{A UPO SASProfile}

A ontologia SASProfile fornece uma estrutura fixa para armazenamento das informações dos usuários e dos conteúdos acessados. A classe User contém dados do usuário, como identificador (ID) e nome (name). A classe Access armazena informações sobre os acessos aos recursos, tais como a hora e a localização do usuário. A classe Resource guarda os identificadores para os recursos, a URL e a lista de usuários que acessaram o conteúdo. A subclasse de Resource, chamada de Link, é responsável por armazenar os links entre o recurso e as ontologias de domínio. A Figura 2 apresenta a estrutura de classes da ontologia.

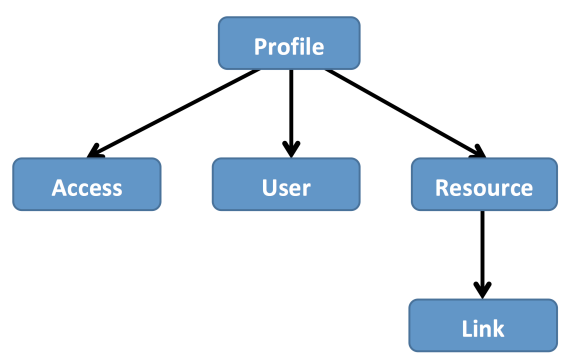

Figura 2. SASProfile - A ontologia de perfil de usuário

Estas classes se relacionam na ontologia através de Ob jectProperties com base no princípio de que os usuários (User) estão ligados (hasAccess) por seus acessos (Access) a um determinado (haslinkedWith) recurso (Resource). Este recurso está relacionado aos itens das ontologias de domínio (hasLinks) através de links (Link). A Figura 3 esquematiza o relacionamento entre as classes no processo de enriquecimento de conteúdo, através das ObjectProperties.

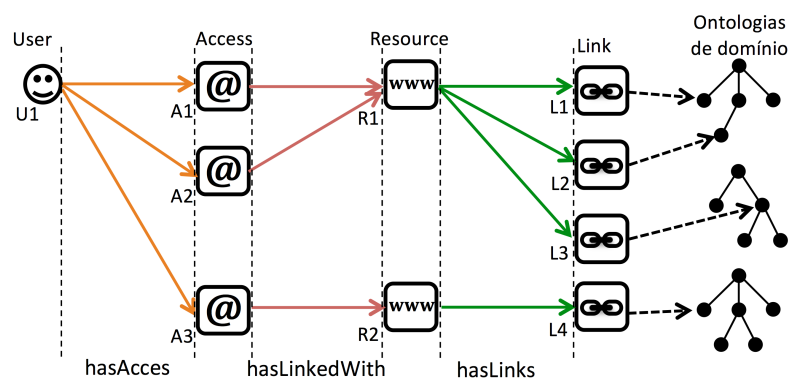

Figura 3. Relacionamento entre as classes através das ObjectProperties

Cada acesso do usuário a um recurso é contabilizado através das instâncias de Access de maneira individual. Na Figura 3, o usuário U1 realiza dois acessos, A1 e 
$\mathrm{A} 2$, ao recurso R1. Os Resources podem conter vários Links para os termos das ontologias de domínio. Neste esquema, o recurso R1 possui três Links para termos de domínio. Estas ligações são estabelecidas pelo Analisador Semântico.

\subsection{Analisador Semântico}

O Analisador Semântico executa o processo de enriquecimento semântico, que é a ação de vincular conceitos semânticos, provenientes de ontologias, a partes de um texto. Para isto, recebe as solicitações de enriquecimento em conteúdos diversos, como textos dos usuários, dados de vídeos do youtube, e quaisquer outras páginas da Web que possam ser indexadas através de uma URL ou URI ${ }^{3}$.

No processo de enriquecimento semântico, as ontologias de domínio são processadas. Como resultado, são produzidas listas contendo os conceitos das mesmas e seus identificadores. Estes conceitos são buscados no conteúdo do recurso. Quando uma correspondência é encontrada, uma anotação é criada. São armazenadas nestas anotações as seguintes informações: o termo do conceito da ontologia, seu identificador único e dados contextuais da correspondência (i.e. o tipo da palavra e a frase em que se encontra). Esta operação é repetida para cada ontologia de domínio cadastrada. As anotações geradas pela análise semântica são armazenadas em estruturas de dados do tipo dicionário, com formação chave $\rightarrow$ valor. Este dicionário é enviado para o Indexador Semântico, que irá tratar as anotações e armazená-las na SASProfile.

\subsection{Indexador Semântico}

O papel do Indexador Semântico é montar e atualizar os perfis dos usuários. Estas atividades compreendem a execução dos algoritmos de seleção de anotações e definição do domínio do recurso, execução das estratégias de armazenamento e captura dos dados na UPO e, por fim, o cálculo das relações entre um recurso e um domínio e do interesse do usuário em um domínio. No processo de indexação de conteúdo, utiliza o dicionário recebido do Analisador Semântico para atualizar os Links e adicionar as ObjectProperties hasAccess, hasLinkedWithe hasLinks aos novos itens.

O Indexador Semântico utiliza a SASProfile juntamente com as ontologias de domínio na determinação dos índices de interesse dos usuários e dos níveis de relação de domínio. No cálculo da relação de domínio, conceitos (classes ou instâncias) vizinhos ao examinado na busca da relação são ponderados no cálculo. Esta ponderação é dada pela similaridade semântica entre os termos. Os seguintes fatores de similaridade são considerados para que o recurso seja contabilizado como sendo parte do conceito analisado:

1. Se o Link aponta para uma classe de domínio: todas as classes derivadas e as classes mães são consideradas similares, assim como todas as instâncias filhas da classe apontada;

2. Se o Link aponta para uma instância de domínio: a classe mãe e toda sua ascendência são consideradas similares, assim como todas as instâncias irmãs diretas da instância apontada.

\footnotetext{
${ }^{3}$ Unique Resource Identifier: um identificador único para um recurso da ontologia, análogo a uma URL.
} 
O percentual dos Links de um recurso que apontam para o domínio analisado e a quantidade percentual dos conceitos da ontologia de domínio contemplados por estes Links são considerados. A relação entre um conteúdo e um domínio específico, Relação de Domínio $\left(R_{d}\right)$, é estabelecida pela quantidade de Links para o domínio de interesse $\left(L_{d}\right)$, quantidade total de Links que um recurso tem $\left(L_{r}\right)$, a quantidade de conceitos mapeados na ontologia de domínio $\left(R_{u}\right)$ e a quantidade total de conceitos mapeáveis na ontologia $\left(R_{t}\right)$, conforme a Equação 1.

$$
R_{d}=\frac{L_{d} / L_{r}+R_{u} / R_{t}}{2}
$$

O interesse do usuário pondera os conteúdos acessados e as frequências de acesso. O cálculo do índice de Interesse do Usuário $\left(U_{i}\right)$ considera a quantidade de acessos registrados para o recurso $\left(A_{d}\right)$, a quantidade total de acessos a todos os recursos $\left(A_{t}\right)$, o somatório das relações de domínio $\left(R_{d}\right)$ e a quantidade de Links para a ontologia de domínio $(d)$, conforme estabelecido na Equação 2.

$$
U_{i}=\left(\frac{A_{d}}{A_{t}}+\frac{\sum_{1}^{d} R_{d}}{d}\right) / 2
$$

A contabilização dos acessos aos recursos $\left(A_{d}\right)$ é baseada nas mesmas características de similaridade empregadas na verificação dos domínios semelhantes. Se o usuário não acessou o recurso que está sendo analisado mas acessou um semelhante, este último será contabilizado como sendo o analisado.

\section{Resultados Experimentais}

No processo de validação do modelo proposto de enriquecimento semântico foram realizados dois experimentos, vinculados a uma aplicação para Aprendizagem Ubíqua que monitora os acessos a sites pelos usuários e recomenda conteúdos voltados para sua aprendizagem, o MobiLE+: (i) verificação da precisão do sistema de análise semântica e (ii) verificação da precisão na demarcação dos perfis de usuários.

Para suporte ao processo de validação, foram realizados o enriquecimento de 240 recursos (sites diversos), divididos igualmente entre três grupos de interesse (estruturas de dados, diabetes e assuntos diversos), sendo pseudoaleatoriamente acessados por 120 usuários fictícios, com 40 acessos para cada.

Na etapa $(i)$, a verificação da precisão foi realizada considerando a correspondência entre os termos presentes nos recursos com os conceitos da ontologia. Na contabilização da precisão, espera-se que a ontologia DataStructures seja aplicada nas anotações de sites com conteúdo sobre estruturas de dados, enquanto a ontologia OntoHealth contemple anotações de sites que tratam sobre diabetes e os sites sobre assuntos diversos não sejam anotados com conceitos de nenhuma das duas ontologias.

Na etapa (ii), a precisão no cálculo do índice de interesse do usuário $U_{i}$ foi analisada sob dois aspectos. No primeiro momento foi realizada uma comparação dos valores de $U_{i}$ baseados apenas nas quantidades de acessos, se $90 \%$ dos acessos são para sites sobre diabetes então o usuário tem $90 \%$ de interesse em diabetes, por exemplo. No segundo 
momento, esta análise foi realizada com base em índices determinados por especialistas nos domínios empregados.

Foram empregadas duas ontologias de domínio, uma que define estruturas de dados na área de algoritmos, a DataStructures, e outra que define a doença diabetes, a OntoHealth. Estas foram desenvolvidas com foco em busca semântica e contêm definições genéricas dos termos característicos do domínio.

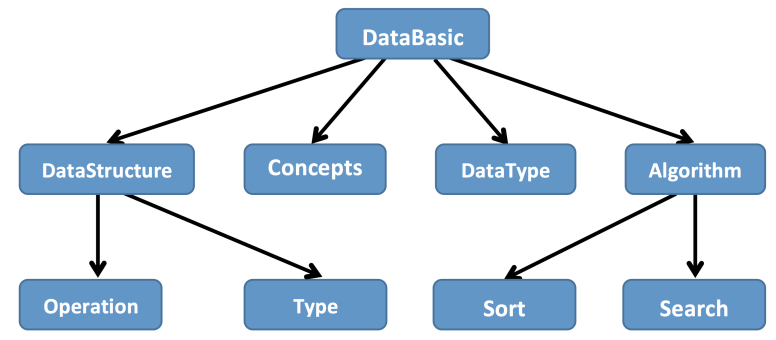

Figura 4. Estrutura de classes da ontologia DataStructures

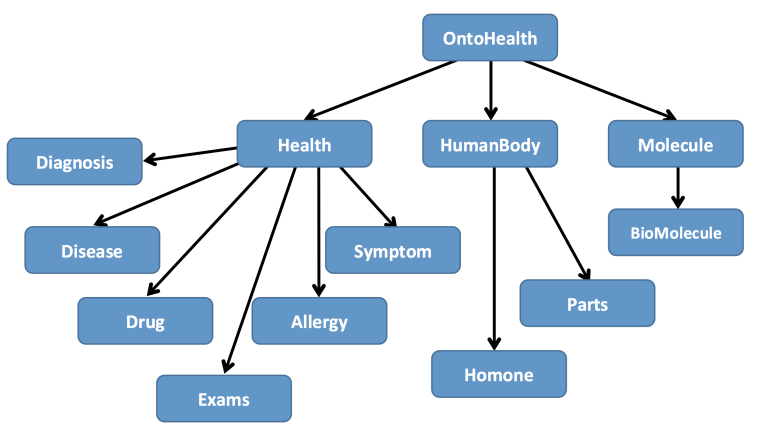

Figura 5. Estrutura de classes da ontologia OntoHealth

A OntoHealth foi desenvolvida por pesquisadores integrantes do projeto MobiLEHealth [Mendes Neto et al. 2014]. A DataStructures foi desenvolvida com o único propósito de validação deste trabalho. As estruturas de classes das ontologias podem ser verificadas nas Figuras 4 e 5.

\subsection{Análise dos Resultados}

Na primeira etapa foi realizada a verificação da precisão do sistema de análise semântica. Após o processamento, um total de $\mathbf{2 2 . 1 0 8} \mathrm{Links}$ foram criados e avaliados individualmente. A Tabela 1 apresenta a quantidade de Links produzidos para cada domínio e distribuídos pelas duas ontologias. Todos os Links que ligaram as ontologias de domínio com sites sobre assuntos diversos foram considerados incorretos. Links que ligaram sites sobre estruturas de dados à ontologia OntoHealth e os que ligaram sites sobre diabetes à ontologia DataStructures foram igualmente considerados incorretos.

Na segunda etapa da validação, um gerador de acessos pseudoaleatório foi aplicado com o objetivo de criar um conjunto de acessos para usuários hipotéticos, povoando a SASProfile com o histórico gerado. Os usuários foram divididos igualmente entre os três grupos de interesse: estruturas de dados, diabetes e assuntos diversos. Os acessos para 
Tabela 1. Distribuição dos Links produzidos

\begin{tabular}{|l|c|c|c|c|c|}
\hline \multirow{2}{*}{ Sites } & \multicolumn{3}{|c|}{ Distribuição dos Links } & \multirow{2}{*}{ Total } \\
\cline { 2 - 5 } & \multicolumn{2}{|c|}{ OntoHealth } & \multicolumn{2}{|c|}{ DataStructures } & \multirow{2}{*}{ Q } \\
\cline { 2 - 5 } & Qtde & $(\%)$ & Qtde & $(\%)$ & \\
\hline Estrutura de Dados & 63 & 0,5 & 12.436 & 99,5 & 12.499 \\
\hline Diabetes & 8.770 & 94,64 & 497 & 5,36 & 9.267 \\
\hline Assuntos Diversos & 117 & 34,21 & 225 & 65,79 & 342 \\
\hline
\end{tabular}

os conteúdos do grupo do usuário foram sorteados com percentuais entre $50 \%$ e $100 \%$, definidos aleatoriamente. O percentual restante dos acessos foi aleatoriamente distribuído entre os conteúdos dos demais grupos.

A partir do histórico de acessos criado, os valores de $U_{i}$ foram calculados. A Tabela 2 apresenta a média dos valores de $U_{i}$ obtidos a partir da distribuição percentual dos acessos e os calculados a partir dos conteúdos analisados pelo SAS para os grupos de interesse "estruturas de dados" e "diabetes". O grupo de interesse para "assuntos diversos" não é contabilizado por não possuir ontologia que defina o domínio. Verifica-se que o desvio padrão para os valores calculados pelo SAS é menor e espera-se que ocorra pelo fato de que, ao considerar o conteúdo e não apenas o tipo do site, a demarcação se torna mais uniforme. O grupo de interesse para "diabetes" apresenta um desvio padrão maior que o de "estruturas de dados". Este fenômeno ocorre devido ao fato de que ao ser menos especializada, e consequentemente menos precisa, a ontologia DataStructures é mais utilizada em marcações incorretas (ver Tabela 1) em conteúdos sobre diabetes, impactando no índice $R_{d}$ e, consequentemente no $U_{i}$.

Tabela 2. Precisão de determinação de Perfis pelo SAS

\begin{tabular}{|c|c|c|c|c|}
\hline \multirow{2}{*}{$\begin{array}{c}\text { Perfis } \\
\text { para os } \\
\text { domínios }\end{array}$} & \multicolumn{2}{|c|}{$U_{i} s$ por Acessos } & \multicolumn{2}{c|}{$U_{i} s$ por SAS } \\
\cline { 2 - 5 } & $\begin{array}{c}\text { Média } \\
\text { Aritmética }\end{array}$ & $\begin{array}{c}\text { Desvio } \\
\text { Padrão }\end{array}$ & $\begin{array}{c}\text { Média } \\
\text { Aritmética }\end{array}$ & $\begin{array}{c}\text { Desvio } \\
\text { Padrão }\end{array}$ \\
\hline Estrutura de Dados & 77,13 & 13,66 & 70,75 & 3,76 \\
\hline Diabetes & 76,44 & 12,71 & 65,59 & 5,22 \\
\hline
\end{tabular}

Os valores de $U_{i}$ calculados a partir do SAS apresentam relações inferiores às determinadas apenas pelas quantidades de acessos por considerarem o teor dos conteúdos, que não abrangem $100 \%$ do domínio relacionado. Para estabelecer a precisão, os sites sobre diabetes e estruturas de dados foram avaliados individualmente por dez especialistas em suas áreas e pontuados conforme sua adequação e abordagem no respectivo domínio. O nível de interesse do usuário $\left(U_{i p}\right)$ foi estabelecido através de uma média ponderada da quantidade de acessos $\left(A_{S}\right)$ e as pontuações (pesos) dadas aos sites $\left(P_{S}\right)$, seguindo as mesmas regras de similaridade apresentadas para o SAS.

Os índices obtidos pelas análises dos especialistas foram considerados padrão. Quaisquer diferenças para mais ou para menos entre estes e os índices do SAS foram consideradas erros. A Figura 6 apresenta os erros obtidos entre os índices calculados pelo SAS $\left(U_{i}\right)$ e os estabelecidos pela análise dos especialistas $\left(U_{i p}\right)$. Observa-se que os erros para os perfis relacionados a estruturas de dados (Figura 6(a))são maiores que os apresentados nos perfis para diabetes (Figura 6(b)). 


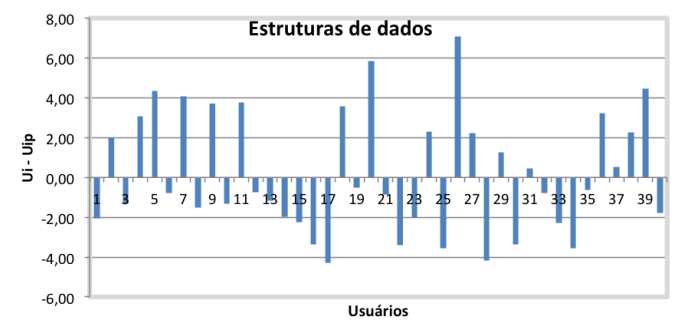

(a)

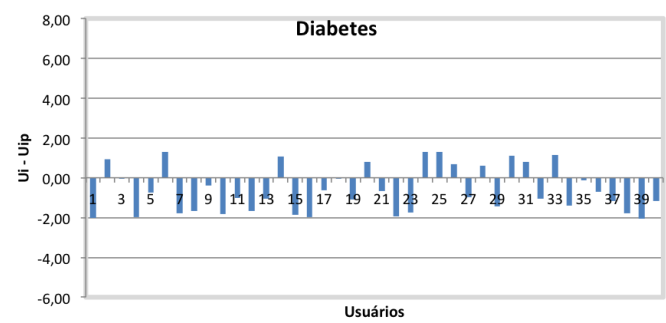

(b)

Figura 6. Diferenças entre $U_{i}$ e $U_{i p}$ para os grupos de usuários

O Analisador Semântico não considera dados contextuais no processo de marcação semântica. Muitos recursos analisados sobre diabetes apresentaram poucas palavras-chaves compatíveis com os conceitos descritos nas ontologias de domínio, porém contendo descrição detalhada do domínio abordado. Este detalhamento foi percebido e pontuado pelo especialista e o resultado pode ser analisado na Figura 6(b): a maioria dos erros foram negativos, ou seja, a relação com o domínio estabelecida pelo especialista foi maior que a encontrada pelo SAS.

\section{Conclusões e Trabalhos Futuros}

O conhecimento do perfil do usuário é fundamental para a personalização do conteúdo apresentado em ambientes de aprendizagem ubíqua. Este trabalho apresentou uma nova abordagem para captura do perfil com base nos conteúdos acessados e também gerados pelo estudante. Ferramentas de processamento de linguagem natural, manipulação de ontologias e construção de perfis de usuários foram empregadas.

Em pesquisas futuras deve-se aplicar a biblioteca no ambiente de aprendizagem ubíqua, MobiLE+; a utilização de um banco de dados semântico para impor mais eficiência no processo de indexação semântica; por fim, uma nova versão do Analisador Semântico que considere o contexto dos termos encontrados e possa realizar um resumo sobre os principais tópicos abordados no recurso.

\section{Referências}

Dspotakis, D., Thakker, D., Lau, L., and Dimitrova, V. (2011). Capturing the semantics of individual viewpoints on social signals in interpersonal communication. In Journal of Web Semantics, Special Issue on Personal and Social Semantic Web.

Ferreira, L. G., Gluz, J. C., and Barbosa, J. L. V. (2012). Um modelo multiagente para recomendação de conteúdo educacional em um ambiente ubíquo. In Anais do $23^{\circ}$ Simpósio Brasileiro de Informática na Educação (SBIE 2012), pages 26-30, Rio de Janeiro, RJ. ISSN 2316-6533.

Gao, Q., Xi, S. M., and Cho, Y. I. (2013). A multi-agent personalized ontology profile based user preference profile construction method. In Robotics (ISR), 2013 44th International Symposium on, pages 1-4.

Gluz, J. C. and Vicari, R. M. (2010). Milos: Infraestrutura de agentes para suporte a objetos de aprendizagem obaa. In XXI Simpósio Brasileiro de Informática na Educação, SBIE, João Pessoa, PB. 
Jácome Jr, L. (2014). Mobile+: Um ambiente de suporte à aprendizagem ubíqua. Master's thesis, Programa de Pós-Graduação em Ciência da Computação, UERN-UFERSA.

Karanasios, S., Thakker, D., Lau, L., Allen, D., Dimitrova, V., and Norman, A. (2013). Making sense of digital traces: An activity theory driven ontological approach. In Journal of the American Society for Information Science and Technology (JASIST).

Mendes Neto, F. M., Costa, A. A. L., Sombra, E. L., Moreira, J. D. C., Samper, J. J., Valentin, R. A. M., Nascimento, R. P. C., and Flores, C. D. (2014). An approach for recommending personalized contents for homecare users in the context of health 2.0. In 7th Euro American Conference on Telematics and Information Systems (EATIS).

Peña, P., del Hoyo, R., Vea-Murguia, J., Gonzalez, C., and Mayo, S. (2013). Collective knowledge ontology user profiling for twitter - automatic user profiling. In Web Intelligence (WI) and Intelligent Agent Technologies (IAT), 2013 IEEE/WIC/ACM International Joint Conferences on, volume 1, pages 439-444.

Reformat, M. and Golmohammadi, S. (2009). Updating user profile using ontology-based semantic similarity. In Fuzzy Systems, 2009. FUZZ-IEEE 2009. IEEE International Conference on, pages 1062-1067.

Rungworawut, W. and Kachonsri, S. (2012). Applying ontology-based personal profile for product search system. In Information Science and Applications (ICISA), 2012 International Conference on, pages 1-5.

So, C. F., Lai, C., and Lau, R. (2009). Ontological user profiling and language modeling for personalized information services. In e-Business Engineering, 2009. ICEBE '09. IEEE International Conference on, pages 559-564.

Sutterer, M., Droegehorn, O., and David, K. (2008). Upos: User profile ontology with situation-dependent preferences support. In Advances in Computer-Human Interaction, 2008 First International Conference on, pages 230-235.

Taraghi, B. (2012). Ubiquitous personal learning environment (uple). In Interactive Collaborative Learning (ICL), 2012 15th International Conference on, pages 1-8.

Vigneshwari, S. and Aramudhan, M. (2012). A novel approach for personalizing the web using user profiling ontologies. In Advanced Computing (ICoAC), 2012 Fourth International Conference on, pages 1-4.

Yarandi, M., Tawil, A., Jahankhani, H., and Hosseini, S. (2012). Ontology-based learner modelling for supporting personalised e-learning. In Interactive Mobile and Computer Aided Learning (IMCL), 2012 International Conference on, pages 113-118.

Yau, J.-K. and Joy, M. (2010). A context-aware personalized m-learning application based on m-learning preferences. In Wireless, Mobile and Ubiquitous Technologies in Education (WMUTE), 2010 6th IEEE International Conference on, pages 11-18.

Yingchen, X., Junzhong, G., Jing, Y., and Zhengyong, Z. (2009). An ontology-based approach for mobile personalized recommendation. In Services Science, Management and Engineering, 2009. SSME '09. IITA International Conference on, pages 336-339. 